\title{
VALIDITY OF C-REACTIVE PROTEIN (CRP) FOR DIAGNOSIS OF NEONATAL SEPSIS IN COMPARISON WITH BLOOD CULTURE
}

\author{
Maria Joseph1, Darly Saramma Mammen², Jayaprakash Parameswaran³, Suresh Sebastian Vadakkedam 4 \\ 1 Junior Resident, Department of Paediatrics, Government Medical College, Kottayam, Kerala, India. \\ ${ }^{2}$ Associate Professor, Department of Paediatrics, Government Medical College, Kottayam, Kerala, India. \\ ${ }^{3}$ Assistant Professor, Department of Paediatrics, Government Medical College, Kottayam, Kerala, India. \\ ${ }^{4}$ Assistant Professor, Department of Paediatrics, Government Medical College, Kottayam, Kerala, India.
}

ABSTRACT

\section{BACKGROUND}

Sepsis is a recognised cause of morbidity and mortality in the new-borns in the developing countries. Sepsis is well-defined as a "clinical syndrome characterised by systemic signs/ symptoms and bacteraemia during the $1^{\text {st }}$ month of life." Neonatal sepsis, a serious illness but curable if identified early. Non-specific signs/ symptoms make it very challenging to formulate a timely clinical diagnosis. Neonatal physicians are looking for a test that would help in neonatal sepsis diagnosis, quickly confirming it and decisively ruling it out. Diagnostic tests like blood cultures are time consuming, so correct diagnosis gets delayed. CRP is very sensitive in detecting negative cases of neonatal sepsis. It is simple, easy and gives early results.

Aim: To find out validity of C-reactive protein in the diagnosis of neonatal sepsis in comparison with blood culture in terms of-

1. Sensitivity, 2. Specificity, 3. Positive predictive value and 4. Negative predictive value.

\section{MATERIALS AND METHODS}

An observational (validation) study was conducted in the NICU and SCNU at Institute of Child Health, Government Medical College, Kottayam to assess validity of CRP for diagnosis of neonatal sepsis in comparison with blood culture. After taking informed consent from parents and permission from Ethical Committee, neonates brought to neonatology unit were selected by purposive sampling technique. Sample size was 90. All neonates having suspected neonatal sepsis were included. Suspected neonatal sepsis was considered if neonates had features of perinatal risk factors, i.e. maternal pyrexia (within 1 week prenatal and/ or 48 hours postnatal), prolonged rupture of membranes (18 hours), foul smelling vaginal discharge or maternal urinary tract infection in last month. Neonates having unexplained hypothermia/ hyperthermia, lethargy, irritability, poor feeding, respiratory dysfunctionapnoea ( $>10$ secs), tachypnoea ( $>60$ breaths/minute), cardiovascular dysfunction- persistent tachycardia ( $>160$ beats/min) or bradycardia ( $<100$ beats/min), poor peripheral circulation, hypotonia or circumoral cyanosis or pallor were also included. Babies with birth asphyxia, birth weight $<1500$ grams, $<32$ weeks gestation and who already had taken antibiotics were excluded. All neonates included in the study were started on empirical antibiotics after drawing samples for blood cultures and CRP. CRP was read as negative when less than $10 \mathrm{mg} / \mathrm{dL}$ and positive when level was more than $10 \mathrm{mg} / \mathrm{dL}$. Blood culture was followed for growth for 7 days. Data collection tool was a pre-tested proforma. Analysis was done using SPSS 23.

\section{RESULTS}

The analysis included a total number of 100 infants. Data was analysed by SPSS version 23 . The baseline characteristics of the study group were comparable in terms of birth weight, gestational age, postnatal age, type of delivery and gender. In this study, CRP was compared to blood culture and was found to have good validity with sensitivity $72.27 \%$, specificity $74.15 \%$, positive likelihoo d ratio 2.75 , negative likelihood ratio 0.37 , positive predictive value $25 \%$ and negative predictive value $95 \%$.

\section{CONCLUSION}

This study proves that CRP is a test with good validity. It can be used for diagnosis of neonatal sepsis and treatment can be initiated before the blood culture results. Neonatal factors like sex, postnatal age, mode of delivery and birth weight do not have statistically significant association with CRP values.

\section{KEY WORDS}

Neonatal Sepsis, Blood Culture, CRP.

HOW TO CITE THIS ARTICLE: Joseph M, Mammen DS, Parameswaran J, et al. Validity of C-reactive protein (CRP) for diagnosis of neonatal sepsis in comparison with blood culture. J. Evolution Med. Dent. Sci. 2018;7(41):4440-4444, DOI: $10.14260 /$ jemds/2018/991

'Financial or Other Competing Interest': None.

Submission 10-08-2018, Peer Review 22-09-2018

Acceptance 29-09-2018, Published 08-10-2018.

Corresponding Author:

Darly Saramma Mammen,

Associate Professor, Department of Paediatrics,

Institute of Child Health,

Government Medical College,

Kottayam-686561, Kerala, India.

E-mail: drdarlymammen@gmail.com

DOI: $10.14260 /$ jemds $/ 2018 / 991$

\section{(c) (i) $(9)$}

\section{BACKGROUND}

Sepsis is a recognised cause of morbidity and mortality in the new-borns in the developing countries. Sepsis is well-defined as a "clinical syndrome characterised by systemic signs/symptoms and bacteraemia during the $1^{\text {st }}$ month of life." ${ }^{1}$ Sepsis is known as 'early onset' disease if present during 1 st 3 days of life and considered as 'late onset' if it follows after 3 days. Of new-borns with early-onset sepsis $85 \%$ present within 24 hours, $5 \%$ present at 24 - 48 hours and a smaller percentage present within 48 - 72 hours. Onset is most rapid in premature neonates.

Early-onset sepsis is associated with acquisition of microorganisms from the mother. Transplacental infection or 
an ascending infection from the cervix may be caused by organisms that colonise the mother's Genitourinary (GU) tract. The neonate acquires the microorganisms, as it passes through the colonised birth canal at delivery. 2,3

\section{Aims and Objectives}

To find out validity of C-reactive protein in the diagnosis of neonatal sepsis in comparison with blood culture in terms of-

- Sensitivity.

- Specificity.

- Positive predictive value.

- Negative predictive value.

\section{MATERIALS AND METHODS}

Study Design

Observational (Validation) study.

\section{Duration of the Study}

June 2016 to June 2017

\section{Study Location}

Department of Paediatrics Medical College, Kottayam.

$$
\begin{aligned}
& \text { Sample Size } \\
& \mathrm{N}(\mathrm{Sp})=\mathrm{TP}+\mathrm{FN} \\
& =\quad \text { Zax Sensitivity (1- Sensitivity) } \\
& \mathrm{Za}=1.96 \\
& \mathrm{Za}^{2}=3.84 \\
& \text { Sensitivity }=76.92
\end{aligned}
$$

Based on a study by Hisamuddin et al on validity of CRP for diagnosis of neonatal reports-

$$
\mathrm{P}, \text { Prevalence }=30 / 1000
$$

(National neonatal and perinatal database)

$\mathrm{N}(\mathrm{SP})=\mathrm{TP}+\mathrm{FN}$

$$
\begin{aligned}
& =\frac{3.84 \times 76.92 \times 23.08}{25 \times 3} \\
& =90.0 \sim 90
\end{aligned}
$$

Minimum sample size required for the study, 90 cases of neonatal sepsis.

\section{Inclusion Criteria}

All babies from 0 to 28 days of life having suspected neonatal sepsis are included in the study.

Suspected neonatal sepsis is considered-

1. Presence of perinatal risk factors, i.e.

A. Maternal pyrexia (within 1 week prenatal and/or 48 hours postnatal).

B. Prolonged rupture of membranes (18 hours).

C. Foul smelling vaginal discharge or/and maternal urinary tract infection diagnosed in last month.

2. Neonates with-

- Unexplained hypothermia/ hyperthermia, lethargy, irritability, poor feeding or milk intolerance.

- $\quad$ Respiratory dysfunction evidenced by apnoea $(>10$ secs), tachypnoea ( $>60$ breaths/minute).
- Cardiovascular dysfunction such as persistent tachycardia ( $>160$ beats $/ \mathrm{min})$ or bradycardia $(<100$ beats/min), poor peripheral circulation, hypotonia or circumoral cyanosis or pallor.

\section{Exclusion Criteria}

Babies who had Suffered-

1. Birth asphyxia.

2. Very low birth weight $<1500$ grams.

3. Extremely premature $<32$ weeks gestation.

4. Neonates who already had taken antibiotics.

5. Maternal history of antibiotic treatment within 1 week prior to delivery.

\section{Study Tools}

Study questionnaire.

\section{Methodology in Brief/ Data Entry and Analysis}

SPSS version 23 was used for statistical analysis of the collected data. Mean and standard deviation were calculated for numerical variables, i.e. age and weight of baby. Frequency and percentages were presented for categorical variables, i.e. qualitative CRP and blood culture results. Sensitivity, specificity, negative and positive predictive values for CRP in identifying babies with culture proven neonatal sepsis were also calculated. Test used was Pearson Chi-square.

This study was designed with an objective to find out the validity of CRP in the diagnosis of neonatal sepsis.

\section{RESULTS}

A total of 120 babies were studied who satisfied the inclusion criteria and ruling out those meeting exclusion criteria. $20 \mathrm{did}$ not give consent, hence were excluded from the study. The analysis included a total number of 100 infants.

\section{Comparison of CRP Results with Blood Culture Results}

\begin{tabular}{|c|c|c|}
\hline & $\begin{array}{c}\text { Blood Culture } \\
\text { Positive }\end{array}$ & $\begin{array}{c}\text { Blood Culture } \\
\text { Sterile }\end{array}$ \\
\hline CRP Positive & $8(\mathrm{~A})$ & $23(\mathrm{~B})$ \\
\hline CRP Negative & $3(\mathrm{C})$ & $66(\mathrm{D})$ \\
\hline \multicolumn{2}{|c|}{ Table 1. CRP and Blood Culture Results } \\
\hline
\end{tabular}

\begin{tabular}{|c|c|c|}
\hline Statistics & Value & $\begin{array}{c}\text { 95\% Confidence } \\
\text { Interval }\end{array}$ \\
\hline Sensitivity & $72.27 \%$ & $52.11 \%$ to $88.21 \%$ \\
\hline Specificity & $74.15 \%$ & $54.11 \%$ to $90.30 \%$ \\
\hline Positive Likelihood Ratio & 2.75 & $1.11 \%$ to $3.52 \%$ \\
\hline Negative Likelihood Ratio & 0.37 & $0.12 \%$ to $0.55 \%$ \\
\hline Disease Prevalence & $31.00 \%$ & $25.22 \%$ to $48.01 \%$ \\
\hline Positive Predictive Value & $75.00 \%$ & 70.02 to $82.31 \%$ \\
\hline Negative Predictive Value & $95.00 \%$ & $78.21 \%$ to $99.00 \%$ \\
\hline \multicolumn{2}{|c|}{ Table 2. Validity values of CRP } \\
\hline \multicolumn{2}{|c}{}
\end{tabular}

CRP has sensitivity of $72.27 \%$ and specificity of $74.15 \%$ compared to blood culture. It has positive likelihood ratio, negative likelihood ratio, positive predictive value and negative predictive value of $2.7 \%, 0.37 \%, 75 \%, 95 \%$ respectively. These values suggest that CRP is a good test compared to blood culture. 


\begin{tabular}{|c|c|c|c|c|}
\hline \multicolumn{2}{|c|}{} & \multicolumn{2}{|c|}{ CRP } & \multirow{2}{*}{ Total } \\
\cline { 3 - 4 } \multicolumn{2}{|c|}{ Age } & Negative & Positive & \\
\cline { 2 - 4 } & Less than 3 days & 28 & 16 & 44 \\
\cline { 2 - 4 } & More than 3 days & 41 & 15 & 56 \\
\hline \multicolumn{2}{|c|}{ Total } & $\mathbf{6 9}$ & $\mathbf{3 1}$ & $\mathbf{1 0 0}$ \\
\hline \multicolumn{2}{|c|}{ Table 3. Comparison of Postnatal Age with CRP } \\
\hline
\end{tabular}

Pearson Chi-square, Odds Ratio for age (less than 3 days/ more than 3 days) 0.499 , $p$ value: 0.157 .

\begin{tabular}{|c|c|c|c|c|}
\hline & \multicolumn{2}{|c|}{ Blood Culture Dicot } & \multirow{2}{*}{ Total } \\
\hline & & Negative & Positive & \\
\hline \multirow{2}{*}{ Age } & $\begin{array}{c}\text { Less than } 3 \\
\text { days }\end{array}$ & 59 & 3 & 62 \\
\hline & $\begin{array}{c}\text { More than } 3 \\
\text { days }\end{array}$ & 30 & 8 & 38 \\
\hline \multicolumn{2}{|r|}{ Total } & 89 & 11 & 100 \\
\hline
\end{tabular}
20.44 .

Pearson Chi-square, Odds ratio $8.167,95 \% \mathrm{CI}-3.263$ to

\begin{tabular}{|c|c|c|c|c|}
\hline \multicolumn{2}{|c|}{} & \multicolumn{2}{c|}{ CRP } & \multirow{2}{*}{ Total } \\
\cline { 3 - 4 } & Male & Negative & Positive & \\
\hline \multirow{2}{*}{ Sex } & Female & 35 & 17 & 52 \\
\cline { 2 - 5 } & 34 & 14 & 48 \\
\hline \multicolumn{2}{|c|}{ Total } & $\mathbf{6 9}$ & $\mathbf{3 1}$ & $\mathbf{1 0 0}$ \\
\hline
\end{tabular}

Table 5. Comparison of Neonatal Sex and CRP Positivity

Pearson Chi-square test value: 0.282 , $\mathrm{p}$ value: 0.584 .

\begin{tabular}{|c|c|c|c|c|}
\hline & \multicolumn{2}{|c|}{ Blood Culture } & \multirow{2}{*}{ Total } \\
\hline & & Negative & Positive & \\
\hline \multirow{2}{*}{ Sex } & Male & 45 & 6 & 51 \\
\hline & Female & 44 & 5 & 49 \\
\hline \multicolumn{2}{|c|}{ Total } & 89 & 11 & 100 \\
\hline
\end{tabular}

Table 6. Comparison of Sex in Blood Culture Positive Cases

Pearson test, Chi-square value: 0.098 and $p$ value: 0.754 . Odds Ratio for sex (Male/ Female) 0.879 with $95 \%$ confidence interval 0.392 to 1.972 .

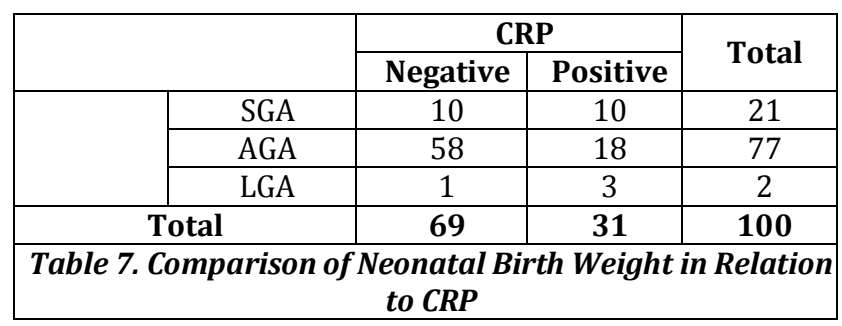

Pearson Chi-square test, Chi-square value: 3.726 , p value: 0.155 .

\begin{tabular}{|c|c|c|c|c|}
\hline \multicolumn{2}{|c|}{} & \multicolumn{2}{c|}{ Blood Culture } & \multirow{2}{*}{ Total } \\
\cline { 3 - 4 } \multicolumn{2}{|c|}{} & Positive & Negative & \\
\hline \multirow{2}{*}{$\begin{array}{c}\text { Birth } \\
\text { Weight } \\
\text { Group }\end{array}$} & SGA & 2 & 37 & 39 \\
\cline { 2 - 4 } & AGA & 8 & 50 & 58 \\
\cline { 2 - 5 } & LGA & 1 & 2 & 3 \\
\hline \multicolumn{2}{|c|}{ Total } & $\mathbf{1 1}$ & $\mathbf{8 9}$ & $\mathbf{1 0 0}$ \\
\hline
\end{tabular}

Table 8. Comparison of Birth Weight with Blood Culture Positivity

Pearson Chi-square value 1.082, p value 0.582 .

\begin{tabular}{|c|c|c|}
\hline & Vaginal & Caesarean Section \\
\hline CRP Positive & 16 & 15 \\
\hline CRP Negative & 45 & 24 \\
\hline \multicolumn{3}{|c|}{ Table 9. Comparison of different Modes of Delivery with } \\
CRP \\
\hline
\end{tabular}

Pearson test, Chi-square value: $1.341, \mathrm{p}$ value: 0.512.

\begin{tabular}{|c|c|c|}
\hline & Vaginal & Caesarean Section \\
\hline $\begin{array}{c}\text { Blood Culture } \\
\text { Positive }\end{array}$ & 7 & 4 \\
\hline Blood Culture Sterile & 59 & 30 \\
\hline \multicolumn{2}{|c|}{ Table 10. Comparison of Delivery with Regard to Blood } \\
Culture
\end{tabular}

Pearson Chi-square test value: 1.639 , p value: 0.802 .

\section{DISCUSSION}

The study was conducted with an aim to find out the validity of CRP in terms of sensitivity, specificity, positive predictive value and negative predictive value on comparing with blood culture which is considered the gold standard. CRP is a simple test. Moreover, it gives quick results. Hence, it can be used for diagnosis and treatment of neonatal sepsis if found to have an acceptable validity. It was a prospective observational study.

The minimum sample size was calculated to be 90 . However, the sample size was increased to 100 neonates to account for the possible loss of data. Thus, a total of 100 babies were studied who satisfied inclusion criteria and ruling out those satisfying the exclusion criteria. All these babies, after taking consent had their blood sampled for CRP and blood culture. Other investigations including haemogram and peripheral smear were also done. Urine studies and CSF studies were done if indicated.

\section{The following Observations were made:}

Baseline characteristics of the study subjects such as the birth weight, gestational age, postnatal age, type of delivery and gender were analysed.

The study showed that the Odds Ratio for age (less than 3 days/ more than 3 days) 0.499 , p value: 0.157 . This was not found to be statistically significant. So the postnatal age in relation to CRP value is not variable. Thus, the CRP values in early onset and late onset sepsis were comparable.

Odds ratio for age (less than 3 days/ more than 3 days) in blood culture positivity was found to be $8.167,95 \%$ CI- 3.263 to 20.44 , p value of 0.442 which was found to be statistically insignificant. So blood culture positivity and postnatal age had no correlation.

The CRP values in relation to neonatal gender was studied. Odds ratio for sex (Male/ Female) was $0.76,95 \%$ CI- 0.292 to 2.030. According to Pearson Chi-square test value: $0.282, \mathrm{p}$ value: 0.584 was found to be statistically insignificant. So CRP was comparable in terms of sex.

The study showed that Odds ratio for sex (Male/Female) was 0.879 with $95 \%$ confidence interval 0.392 to 1.972 in relation to blood culture positivity. P value of 0.754 was found to be statistically insignificant. So sex was not related to blood culture positivity.

Therefore, CRP and blood culture results are comparable with respect to gender and postnatal age. 
Of the 31 neonates with CRP positivity, 16 (51.7\%) were born by vaginal delivery and $15(48.3 \%)$ by caesarean section. Of the 11 neonates with blood culture positivity 7 (63.6\%) and $4(36.4 \%)$ were born by vaginal delivery and caesarean delivery respectively and of the 11 cases with blood culture positivity, 7 (63.6\%) were by vaginal delivery and 4 (36.3\%) were by caesarean section.

In the study, the birth weight distribution of the neonates is as follows. Of the 69 neonates in the CRP positive group, 10 (14.5\%), $58(84 \%)$ and $1(1.45 \%)$ were SGA, AGA, LGA respectively. According to Pearson test, Chi-square value: 3.726 , $p$ value: 0.155 was found to be statistically insignificant. So the birth weight distributions were comparable in terms of CRP results.

Of 11 neonates with blood culture positivity 2 (18.18\%), $8(72.72 \%)$ and 1 (9.06\%) were SGA, AGA and LGA respectively. According to Pearson test, Chi-square value: 1.082 and $p$ value: 0.582 were found to be statistically insignificant. So the birth weight distributions were comparable in terms of blood cultures.

In the study, it was found that Odds ratio for I/T (more than 0.2 / less than 0.2 ) is 5.45 and $p$ value of 0.02 . It is statistically significant. Therefore, the study concludes that a positive I/T ratio of $>0.2$ points towards an increased chance of blood culture positivity (Neonatal sepsis).

On comparing I/T ratio with urine culture positivity, Pearson Chi-square test value: 1.639 and $p$ value: 0.802 were not statistically significant. I/T ratio was not found to have significant relation to urine culture results, hence not useful in diagnosing urinary tract infections.

Comparison of I/T ratio values with CSF culture results in the study showed that Pearson Chi-square test value 2, 7 and $p$ value of 1.2 which is statistically insignificant. Hence, showed that I/T ratio is not sensitive for diagnosis of meningitis.

The primary objective of the study was to find out the validity of CRP test comparing with blood culture, which is considered the gold standard. The following statistical values were drawn-
1. Sensitivity- $72.27 \%$
2. Specificity- $74.15 \%$
3. Positive Likelihood Ratio- 2.75
4. Negative Likelihood Ratio- 0.37
5. Positive Predictive Value- $75 \%$
6. Negative Predictive Value- $95 \%$

A study done by Bhattacharya $\mathrm{K}$, Bandyopadhyay $\mathrm{M}$, Karmakar BC, Battacharya S, Banerjee P, Chatterjee S, Dutta in RG Medical College, Kolkata on blood culture positivity and Creactive protein variability in neonatal septicaemia showed clinical correlation of CRP as significant $(65.2 \%$ of patients has blood culture positivity). It can be used as an important parameter in infants at risk of septicaemia (significant $>6$ $\mathrm{mg} / \mathrm{dL}$ ) and early institution of antimicrobials therapy. It has got prognostic value. ${ }^{4}$

In the double-blind randomised controlled trial by Patil S, Dutta S, Attri SV, Ray P, Kumar P at Institute of Medical Education and Research, Chandigarh, India, evaluated change in CRP from baseline as a diagnostic test for identifying empirical use of sensitive antibiotics. ${ }^{5}$

Another, similar randomised controlled trial study by Bhargava M, Saluja S, Sindhuri U, Saraf A and Sharma P at Sir Gangaram Hospital, New Delhi evaluated serial CRP values in the first $48 \mathrm{hrs}$. of antibiotic therapy help to predict whether the causative organism is sensitive to the empirical antibiotics administered. Inference was that CRP is a highly sensitive and specific predictor of neonatal sepsis. ${ }^{6}$

A study on predictive values of serial C-reactive protein in neonatal sepsis was done by Nuntnarumit $\mathrm{P}$, Pinkaew $\mathrm{O}$ in Ramathibodi Hospital, Mahidol University, Bangkok, Thailand. Inference was serial CRP that showed very high predictive values for diagnosis of neonatal sepsis. The sensitivity, specificity, positive predictive value and negative predictive value of CRP for proven sepsis and localised infection at cut-off point $>$ or $=5 \mathrm{mg} / \mathrm{L}$ were 100 percent, 94 percent, 91.6 percent and 100 percent respectively. ${ }^{7}$

A study was conducted by Hisamuddin E, Hisam A, Wahid $S$ and Raza G for validity of C-reactive protein (CRP) for diagnosis of neonatal sepsis. The sensitivity and specificity of CRP in diagnosis of acute neonatal sepsis was $76.92 \%$ and $53.49 \%$ respectively, while it had a positive predictive value of $80 \%$ and negative predictive value of $48.94 \%$. Overall, the diagnostic accuracy of CRP in diagnosis of neonatal sepsis was $70.07 \%$. Mean age of the neonates was 5.72 days +3.86 . Male patients were $81(55.1 \%)$, while $66(44.9 \%)$ were females. Concluded that CRP estimation does have a role in the diagnosis of neonatal sepsis, but the test is not specific enough to be relied upon as the only indicator. ${ }^{8}$

My study has shown a high sensitivity for CRP of $72.27 \%$. This indicates that the test is a good screening test, meaning CRP negativity can effectively rule out neonatal sepsis. The study also showed a high specificity of $74.15 \%$, which indicates that it is a test comparable to blood culture in diagnosing neonatal sepsis.

The study also showed Odds ratio for I/T (More than 0.2 /less than 0.2 ) 5.45 and $p$ value of 0.02 . It is statistically significant. Therefore, positive I/T ratio points towards an increased chance of blood culture positivity. However, I/T ratio was not found to have significant relation to urine culture results. Pearson Chi-square test value: 1.639 and $p$ value: 0.802 was not statistically significant. Hence, it is not dependable in diagnosing urinary tract infections.

The above statements means that tests like CRP and I/T ratio can be used for early diagnosis of neonatal sepsis. Empirical antibiotics can be started based on these test results as blood culture results are time consuming.

Our study results was comparable to study by Bhattacharya K, Bandyopadhyay M, Karmakar B. C, Battacharya S, Banerjee P, Chatterjee S and Dutta in RG Medical College, Kolkata on blood culture positivity and Creactive protein. The study conducted by Hisamuddin E, Hisam A, Wahid S and Raza G on validity of C-reactive protein (CRP) for diagnosis of neonatal sepsis also showed comparable results. The sensitivity and specificity of CRP in diagnosis of acute neonatal sepsis was $76.92 \%$ and $53.49 \%$ respectively, while it had a positive predictive value of $80 \%$ and negative predictive value of $48.94 \%$. Overall, the diagnostic accuracy of CRP in diagnosis of neonatal sepsis was $70.07 \%$.

From the above discussion, the following observations were made.

\section{CONCLUSION}

This study proves that CRP is a test with good validity. It can be used for diagnosis of neonatal sepsis and treatment can be initiated before the blood culture results are available. 


\section{Jemds.com}

Neonatal factors like sex, postnatal age, mode of delivery and birth weight do not have statistically significant association with CRP values.

\section{Limitations of the Study}

- Standardisation of CRP tests is a matter of concern.

- Serial CRP values were not studied, hence time-based standardisation was not done.

- CRP values after initiation of antibiotics were not analysed. Hence, prognostic implication is unclear.

- Hence, studies on large samples and with longer duration of follow-up should be conducted.

There is a strong need for more randomised controlled trials in future to validate the results of this study.

\section{REFERENCES}

[1] Edwards MS, Baker CJ. Sepsis in the new-born. In: Gershon AA, Hotez PJ, Katz SL, eds. Krugman's infectious diseases of children. $11^{\text {th }}$ edn. Philadelphia, PA: Mosby 2004: p. 545-61.

[2] Schuchat A. Neonatal group B streptococcal diseasescreening and prevention. $\mathrm{N}$ Engl J Med 2000;343(3):209-10.

\section{Original Research Article}

[3] Hornik CP, Fort P, Clark RH, et al. Early and late onset sepsis in very-low-birth-weight infants from a large group of neonatal intensive care units. Early Hum Dev 2012;88(Suppl 2):S69-S74.

[4] Bhattacharyya K, Bandyopadhyay M, Karmakar BC, et al. A study on blood culture positivity and C-reactive protein variability in neonatal septicaemia at neonatal intensive care unit of a tertiary care hospital. J Indian Med Assoc 2012;110(12):920-1, 925.

[5] Patil S, Dutta S, Attri SV, et al. Serial C reactive protein values predict sensitivity of organisms to empirical antibiotics in neonates: a nested case-control study. Arch Dis Child Fetal Neonatal Ed 2016 Apr 29. pii: fetalneonatal-2015-309158.

[6] Bhargava M, Saluja S, Sindhuri U, et al. Elevated mean neutrophil volume+CRP is a highly sensitive and specific predictor of neonatal sepsis. Int J Lab Hematol 2014;36(1):e11-e4.

[7] Nuntnarumit P, Pinkaew 0, Kitiwanwanich S. Predictive values of serial C-reactive protein in neonatal sepsis. J Med Assoc Thai 2002;85(Suppl 4):S1151-8.

[8] Hisamuddin E, Hisam A, Wahid S, et al. Validity of Creactive protein (CRP) for diagnosis of neonatal sepsis. Pakistan J Med Sci 2015;31(3):527-31. 